\title{
How Does Ultrasound Manage Pancreatic Diseases? Ultrasound Findings and Scanning Maneuvers
}

\author{
Shinji Okaniwa
}

Department of Gastroenterology, Iida Municipal Hospital, Iida, Japan

As ultrasound (US) is simple and less invasive than other imaging modalities, this technique is widely used for mass screening. However, visualizing the entire pancreas due to complicated anatomy, obesity and overlying gas can be difficult. US plays a key role in the diagnosis of pancreatic carcinoma (PC), of which tumors smaller than $10 \mathrm{~mm}$ (TS1a) and pancreatic carcinoma in situ are expected to have good prognosis. To detect these forms of PC, main pancreatic duct (MPD) dilatation (3 $\mathrm{mm}$ or more) and pancreatic cysts (5 $\mathrm{mm}$ or larger) are US findings of high-risk individuals (HRIs), and these subjects should be observed periodically. Scanning maneuvers are also important for both screening for PC and follow-up of HRIs. As lesions in the groove area and ventral pancreas do not affect the MPD or extrahepatic bile duct, we should pay attention to these areas. Visualization of the tail is also challenging due to gas and stool in the alimentary tract. As the position of the pancreas changes depending on the body posture, and several different body positions should be employed, such as the right lateral decubitus, sitting, and upright positions, rather than only applying strong compression with the transducer. In cases with poor visualization, the liquid-filled stomach method is highly recommended. (Gut Liver 2020;14:37-46)

Key Words: Ultrasound; Pancreatic carcinoma; Risk factors; Pitfalls; Mass screening

\section{INTRODUCTION}

As ultrasound (US) is simple and less invasive, it is widely used for mass screening and initial imaging for suspected cases of pancreatobiliary diseases in Japan. However, the pancreas is a slender organ located deep inside behind the stomach, and it is sometimes difficult to visualize the entire pancreas due to complicated anatomy, obesity and overlying gas.

Pancreatic carcinoma (PC) is a serious condition. Sensitivity of US in detecting PC is approximately $75 \%$ to $98 \%{ }^{1,2}$ However, tumor detection rates of US and computed tomography (CT) of PC with stage1 (invasive carcinoma with tumor diameter of $<20$ $\mathrm{mm}$ confined within the pancreas, along with the absence of regional lymph node metastasis [NO] and distant metastasis [MO]) are only 67.3\%, 65.8\% respectively, and significantly lower than 92.4\% of endoscopic ultrasonography (EUS). ${ }^{3}$

In order to detect PC with good prognosis, we should clarify risk factors for PC such as a viral infection in hepatocellular carcinoma. In 2016, the Japan Pancreas Society (JPS) published clinical guidelines for $\mathrm{PC}^{4}$ suggesting some factors of high-risk individuals (HRI) (Table 1). Patients with more than one risk factor are recommended to undergo further examinations to detect PC.

As some US findings such as dilated main pancreatic duct (MPD) and pancreatic cysts are also indicative factors of HRI (Table 1), US could contribute to not only pick up HRI, but detect PC with good prognosis by the periodical follow-up with US. ${ }^{5,6}$ To evaluate the entire pancreas in HRI, we should employ a variety of scanning methods, such as changing the patients' body position and the stomach filling method, ${ }^{7}$ and use highresolution transducers with magnified images to detect small lesions and delineate the MPD.

The aims of this review are to summarize the characteristics of PC with good prognosis, important US findings, pitfalls of pancreatic examinations, and practical solutions.

\section{CANDIDATES OF PC WITH GOOD PROGNOSIS}

PC is associated with an extremely poor prognosis, and its 5-year survival rate does not reach $10 \%{ }^{8,9}$ The poor prognosis was a result of delayed diagnosis in the natural course of PC.

\footnotetext{
Correspondence to: Shinji Okaniwa

Department of Gastroenterology, Iida Municipal Hospital, 438 Yawata-machi, Iida 395-8502, Japan

Tel: +81-265-21-1255, Fax: +81-265-21-1266, E-mail: okaniwa@cocoa.ocn.ne.jp

Received on December 21, 2018. Accepted on February 11, 2019. Published online April 24, 2019. pISSN 1976-2283 eISSN 2005-1212 https://doi.org/10.5009/gnl18567

() This is an Open Access article distributed under the terms of the Creative Commons Attribution Non-Commercial License (http://creativecommons.org/licenses/by-nc/4.0) which permits unrestricted non-commercial use, distribution, and reproduction in any medium, provided the original work is properly cited.
} 
Using a mathematical model, Yachida et al. ${ }^{10}$ estimated an average of 11.7 years from the initiating carcinogenesis until development of the parental clone, an average of 6.8 years to the development of metastatic subclones within the primary PC, and an average of 2.7 years until the death of patients. Theoretically, there are a few years to detect PC in early stage.

According to the Japan Pancreatic Cancer Registry, the 5-year survival rates of patients diagnosed with the Union for International Cancer Control (UICC) stage IA and stage IB were 68.7\%, and 59.7\%, respectively. ${ }^{11}$ When the tumor size was $10 \mathrm{~mm}$ or smaller (TS1a), the 5-year survival rate reached $80.4 \%$ and was significantly higher than that of cases with tumor size larger than $10 \mathrm{~mm}$ (TS1b). ${ }^{11}$ According to the latest multicenter study in Japan, the estimated overall survival rates at 10 years after the resection for stage I (TS1a) and stage I (TS1b) were 93.8\%, and 78.9\%, respectively. ${ }^{3}$ Although the difference was not statistically significant, overall survival rates tended to be lower in the stage I (TS1b) cases. ${ }^{3}$

However, in cases where the primary tumor of $10 \mathrm{~mm}$ already has a probability of $28 \%$ of harboring metastases at diagnosis; as the primary tumor size increases to $20 \mathrm{~mm}$ and $30 \mathrm{~mm}$, the risk of harboring metastases increases to 73\% and 94\%, respectively. $^{12}$

These results suggest tumor size $10 \mathrm{~mm}$ or smaller (TS1a)

Table 1. Risk Factors for Pancreatic Carcinoma

\begin{tabular}{ll}
\hline Family history & Pancreatic carcinoma \\
& Hereditary pancreatic carcinoma \\
Accompanying diseases & Diabetes mellitus \\
& Obesity \\
& Chronic pancreatitis \\
& IPMN \\
US findings & MPD dilatation $(\geq 3 \mathrm{~mm})$ \\
& Cystic lesion $(\geq 5 \mathrm{~mm})$ \\
Habits & Smoking \\
& Heavy drinking \\
\hline
\end{tabular}

IPMN, intraductal papillary mucinous neoplasm; US, ultrasound; MPD, main pancreatic duct. could be defined as PC with good prognosis now (Fig. 1). But we should sustain our effort to detect much smaller PC.

\section{PANCREATIC CARCINOMA IN SITU}

PC develops through a stepwise progression from precursor lesions. Hruban et al. ${ }^{13}$ reported a genetic progression model from the precursor lesions named pancreatic intraepithelial neoplasia (PanIN) to PC. According to their progressions, the extent of atypia was classified as PanIN-1 (low-grade dysplasia), PanIN-2 (moderate-grade dysplasia), and PanIN-3 (high-grade dysplasia). Pancreatic carcinoma in situ (PCIS) are classified as PanIN-3.

The 5-year survival rate and estimated overall survival rates at 10 years after the resection of cases with UICC stage 0 (PanIN-3/PCIS) were $85.8 \%{ }^{11}$ and $94.7 \%{ }^{3}$, respectively. However, the corresponding proportion of stage 0 cases accounted for only $1.7 \% .^{11}$

As PCIS usually do not show any formed mass lesions, most PCIS were usually detected by the MPD dilatation (76.5\%), and seldom by the MPD stenosis (5.9\%) and tumor detection (8.8\%). ${ }^{3}$ The number of abnormal findings of US, CT, and EUS in PCIS was significantly lower than that in stage I $(\mathrm{p}<0.01){ }^{3}$

According to some recent pathological research, PCIS is frequently accompanied by localized pancreatitis with infiltration of inflammatory cells, fibrosis, and fatty infiltration in the parenchyma. ${ }^{14-18}$ Abnormal findings of the MPD, such as irregularity, non-continuous narrowing, granular defects, and dilatation were the most important findings observed by endoscopic retrograde cholangiopancreatography. ${ }^{19}$ EUS can detect localized pancreatitis or fatty infiltration around PCIS as a slightly low echoic lesion. ${ }^{16,20}$

These results suggest that we should employ the high-resolution transducer with magnified images and trace the MPD to pick up not only cases with dilated MPD but cases with stenotic MPD accompanied with branch duct dilatation and/or faint hypoechoic area (Fig. 2).
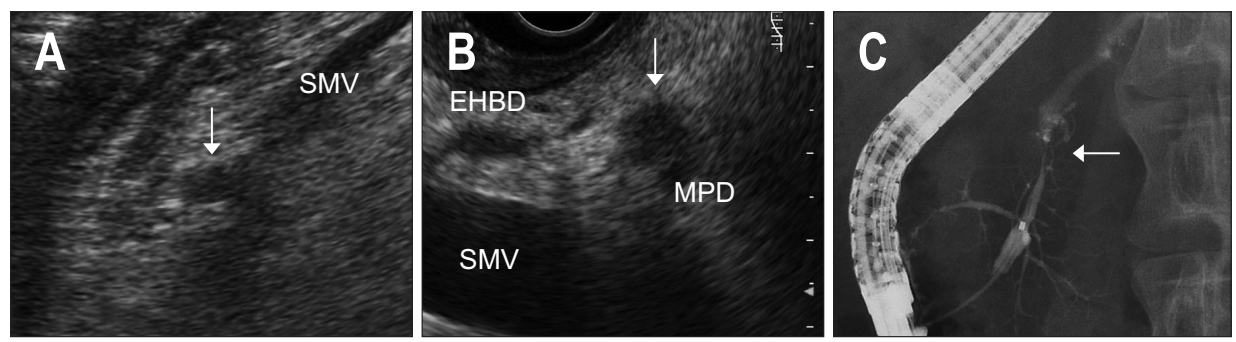

Fig. 1. A case of TS1a in the pancreatic head. (A) Ultrasonography (US) shows a small hypoechoic lesion (arrow) in the head near the superior mesenteric vein (SMV). (B) Endoscopic US (EUS) delineates the small hypoechoic lesion (arrow) with irregular margin and spinous structures attached to the MPD. (C) Endoscopic retrograde pancreatography shows localized stenosis of the MPD (arrow) with dilatation of the distal part. TS1a, tumors smaller than $10 \mathrm{~mm}$; MPD, main pancreatic duct; EHBD, extrahepatic bile duct. 


\section{IMPORTANT US FINDINGS FOR SCREENING}

According to the manual for abdominal ultrasound in cancer screening and health checkups, ${ }^{21}$ solid lesions, cystic lesions, calcifications, MPD dilatation, morphological abnormality (swelling or atrophy), and localized swelling should be detected and evaluated through mass screening.

\section{Solid lesions}

Their number, echogenicity, contour, internal structure, and relationship with MPD are useful US findings for differential diagnoses.

Both PC and pancreatitis result in heterogeneous consistency, returning echoes of lower amplitude than normal. ${ }^{22,23}$ As the tumor grows, PC develops a central hyperechoic area. ${ }^{24}$ The contour is basically clear and irregular reflecting the invasive growth of PC but becomes relatively unclear with involvement of inflammatory changes in many cases. ${ }^{24}$ Spinous structures which correspond to the irregular invasion into the pancreatic parenchyma pathologically, were characteristic and observed in $31.0 \%$ of PC less than $20 \mathrm{~mm} .{ }^{25}$ However, cystic components which correspond to the necrosis were rare. ${ }^{25}$ The presence of MPD dilatation at the caudal part of the lesion, especially accompanied by irregularity of the MPD is a characteristic of $\mathrm{PC}^{24,25}$

\section{Cystic lesions}

The prevalence of pancreatic cysts in asymptomatic individuals in the general population is $2.4 \%$ to $3.5 \%,{ }^{26-28}$ and increases with age and has higher incidence in females. ${ }^{28}$ The location, number, contour, internal structures, internal echo texture, and communication with the MPD are useful findings for differential diagnoses. ${ }^{29}$ The presence of solid components including thickened cystic wall and mural nodule is closely related to the malignant potential of cystic lesions. ${ }^{30}$ Furthermore, whenever pancreatic cysts are encountered in patients of cancer-prone age without a history of pancreatitis, the proximal pancreas should be carefully examined to exclude PC. ${ }^{31}$

Presence of cysts ( $5 \mathrm{~mm}$ or larger) is also recognized as US findings for HRI. Prevalence of pancreatic cysts in asymptom- atic HRI is $38.9 \%$, which is significantly higher than in the general population, and 60.7\% had multiple lesions. ${ }^{32}$ The hazard ratio was $6.23(\mathrm{p}=0.003)$ and the cumulative 5-year risk of PC was high at $1.84 \%$ (95\% confidence interval [CI] 0.88\% to $3.82 \%$ ) in subjects with cysts. ${ }^{6}$ So, periodical follow-up for those subjects is highly recommended for early detection of PC.

\section{Calcifications}

Calcifications inside the MPD could be diagnosed as pancreatolithiasis. As solid pseudopapillary neoplasms and neuroendocrine neoplasms sometimes contain calcification inside lesions, ${ }^{24}$ check the surrounding echotexture to rule out those solid mass lesions.

\section{MPD dilatation}

The incidence of MPD dilatation in the body detected by US in the general population was reported to be not high, at most 1.25\% for $3 \mathrm{~mm}$ or more. ${ }^{33,34}$ The diameter of the MPD was measured between the upper edge of the anterior line and the posterior line of the MPD and rounded off to one decimal place. As the diameter of the MPD may change during the examination, ${ }^{35}$ we should measure the diameter at least twice in the body on the transverse scan with magnified images.

MPD dilatation is a common US finding with PC. The incidence in smaller than $10 \mathrm{~mm}$ (TS1a) and PCIS is 59\% to $74.3 \%{ }^{3,25,36}$ and $76.5 \%$ respectively. ${ }^{3}$ To rule out the occult PC and IPMN, evaluate the presence of stricture of the proximal side or protruded papillary lesions inside the dilated MPD. ${ }^{21}$ To evaluate those findings precisely, the high-resolution transducer with magnified images are essential.

The dilatation of MPD ( $3 \mathrm{~mm}$ or more) is also recognized as US findings for HRI. The hazard ratio was $6.38(\mathrm{p}=0.018)$ and the cumulative 5-year risk of PC was high at 1.84\% (95\% CI, $0.88 \%$ to $3.82 \%$ ) in subjects with MPD dilatation. ${ }^{7}$

Periodical survey for those cases is also recommended for early detection of PC.

\section{Morphological abnormality (swelling or atrophy)}

The size of pancreas is different from individual to individual. The dimensions of the head, body, and tail of the pancreas cor-
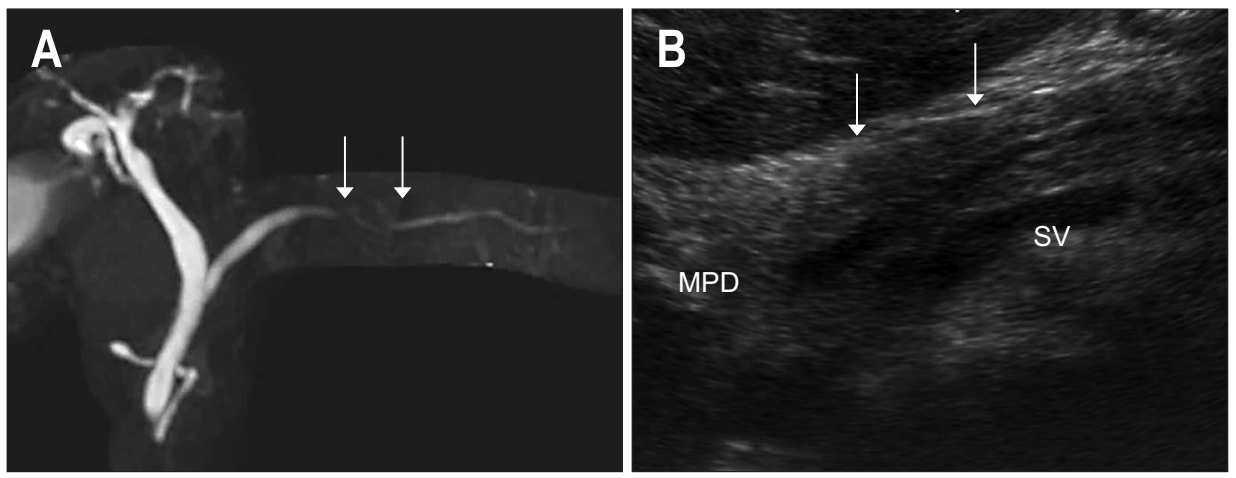

Fig. 2. A case of localized pancreatitis. (A) Magnetic resonance cholangiopancreatography shows localized stenosis (arrows) of the MPD in the body of the pancreas. (B) High-resolution ultrasonography delineates a faint hypoechoic area surrounding the stenotic MPD (arrows).

MPD, main pancreatic duct; SV, splenic vein. 
relate to patient age, weight, height, and body surface area. ${ }^{37}$ Regarding the reference value of each pancreatic part on a transverse section, antero-posterior diameter of the pancreatic head, body and tail are 19-25 mm, 15-20 mm and 20-25 mm, respectively. ${ }^{38}$ During screening, sizes larger than $30 \mathrm{~mm}$ and smaller than $10 \mathrm{~mm}$ could be candidates for detailed examination. $^{21}$

\section{Localized swelling}

In general, a normal pancreas has a similar sized head and tail with a thinner body. Localized swelling is defined as the localized thickness of pancreatic parenchyma with smooth contour. ${ }^{21}$ The most important targets of this finding are PC and autoimmune pancreatitis. In cases of decreasing echogenicity, irregularity of echotexture, and blurriness of MPD inside the swollen area ${ }^{21}$ further examination is highly recommended.

\section{PITFALLS OF PANCREATIC EXAMINATIONS}

The pancreas is usually divided into three parts: head, body and tail. The lower part of the head is called the uncinate process (Fig. 3). ${ }^{39}$

\section{Pancreatic head}

The pancreatic head is larger when examined and measured in the cranio-caudal diameter compared to the other pancreatic diameter. ${ }^{40}$

Common US findings suggesting pancreatic head carcinomas include dilatation of the extrahepatic bile duct (EHBD) and/or MPD. However, PC arising from the uncinate process does not show those findings until very late stage. ${ }^{41}$ As PC in the groove area is also not accompanied by the dilatation of the MPD ${ }^{42}$ we should also pay attention to those areas. Therefore, the marginal area between the duodenums, especially the caudal parts should be delineated to rule out PC.

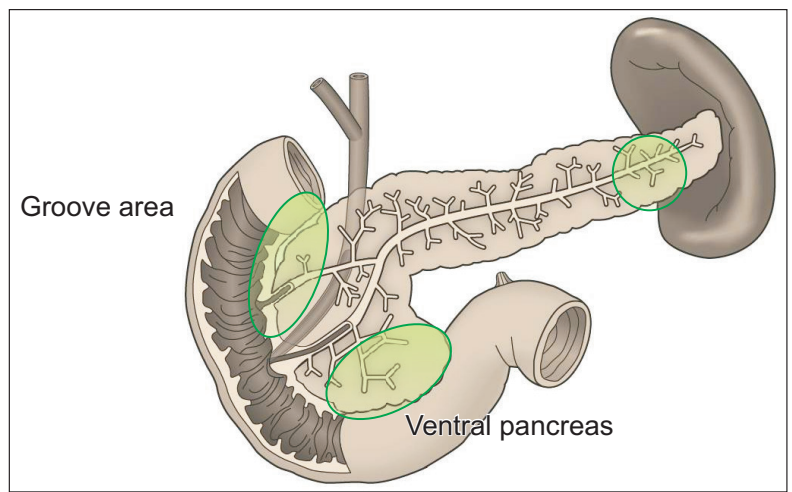

Fig. 3. Pitfalls of pancreatic examinations. Important portions which are generally overlooked by ultrasound (green areas).

\section{Pancreatic tail}

According to the sensitivity in detecting pancreatic cysts (5 $\mathrm{mm}$ and larger) comparing to the MRI, routine US had a sensitivity of 70.2\% for all cysts. ${ }^{7}$ When the sensitivity was examined in parts of the pancreas, routine US had a sensitivity of $74.2 \%$ in the uncinate process, $69.5 \%$ in the head, $81.0 \%$ in the body, $67.0 \%$ in the body-tail, and only $26.7 \%$ in the tail. ${ }^{7}$ According to another study using a CT-fusion image, the pancreatic tail was completely identified in only $33 \%$ by left intercostal scanning in spine. ${ }^{43}$ About $25 \%$ of the tail was unobservable and positively correlated with body mass index and waist circumference. ${ }^{43}$ The limitation in the depiction of the pancreatic tail may be attributed to shadowing from gas in the stomach and stool in the colon, and this part is a main candidate for practical solutions which will be mentioned later.

\section{BASIC SCANNING METHODS}

Transverse and longitudinal upper abdominal sections are used to visualize the pancreas, as well as oblique intercostal and subcostal sections, especially for the head and tail. To obtain the broad range of visualization of the pancreas, start the examination with the patient in supine position, then employ the left lateral decubitus position with suspended inspiration and expiration.

\section{Upper abdominal longitudinal scans}

Place the transducer longitudinally on the upper abdomen, and define the aorta (Ao), celiac artery (CA), superior mesenteric artery (SMA), and splenic vein (SV). Notice the pancreatic body above the CA and SMA, and check the caliber of MPD (Fig. 4a). Move the transducer to the left in parallel steps, and try to define the pancreatic tail and left kidney (LK), adjusting the focus deeper to get good images (Fig. 4b). Return to the Ao and move the transducer to the right side, and define the superior mesenteric vein (SMV). After magnifying the image, delineate the uncinate process which lies posterior to the SMV at this section (Fig. 4c). Move the transducer further right to define the inferior vena cava (IVC), and delineate the pancreatic head and EHBD above the IVC (Fig. 4d). Move the transducer further to the right side to define the descending part of duodenum or gallbladder, and delineate the entire pancreatic head.

As gas moves with the postural change, repeat this tail-tohead pass in both the supine and left lateral decubitus positions. In cases of poor pancreatic head imaging, trace the entire EHBD in left decubitus position (Fig. 5),$^{44}$ then move the transducer parallel from the descending part of the duodenum to the SMV to check the entire pancreatic head.

\section{Upper abdominal transverse scans}

Start scanning from the liver to reduce image degradation 

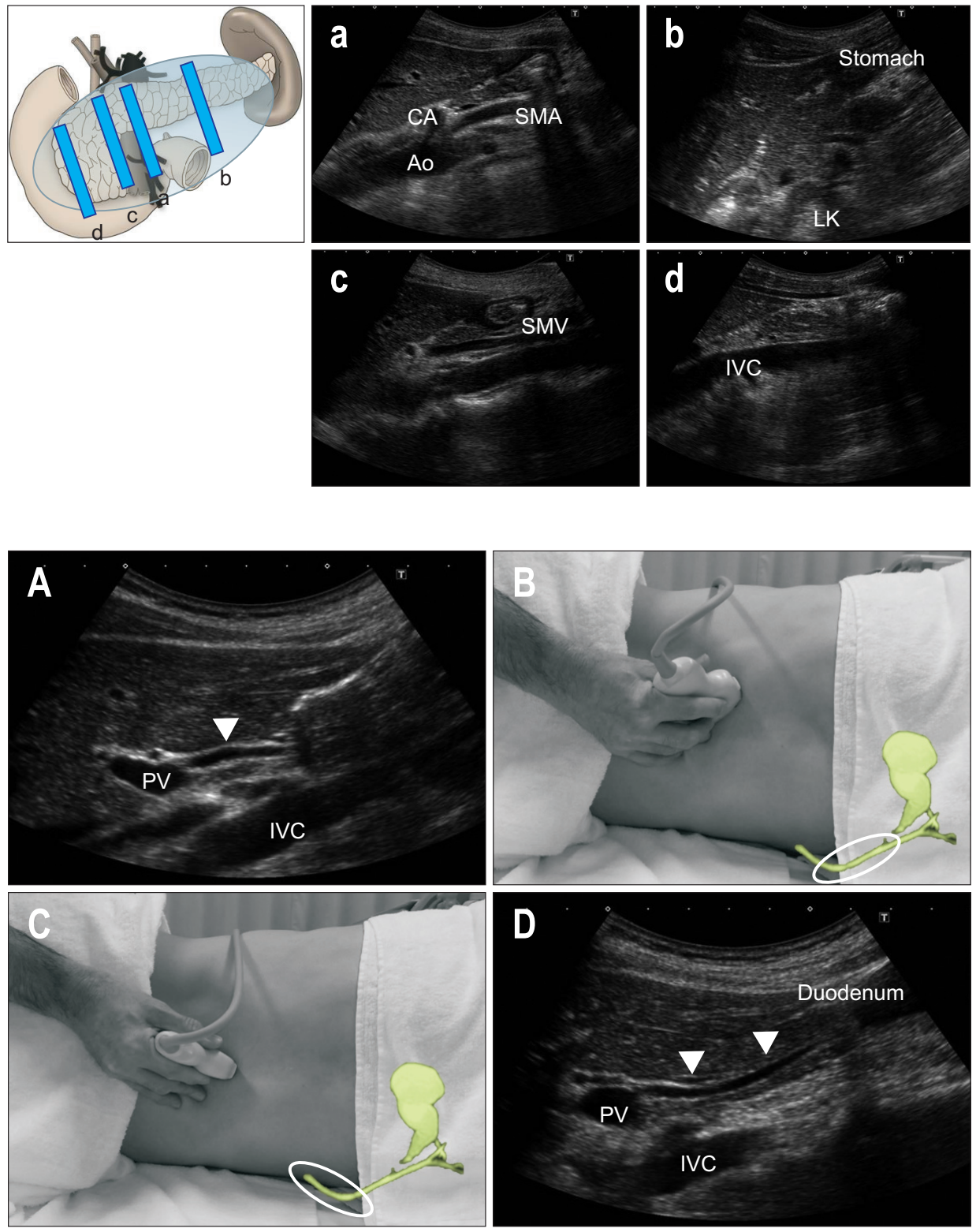

Fig. 4. Upper abdominal longitudinal scans. (a) Notice the pancreatic body above the celiac artery (CA) and superior mesenteric artery (SMA). (b) Move the transducer to the left in parallel steps, and try to define the pancreatic tail and left kidney (LK). (c) After magnifying the image, delineate the uncinate process. which lies posterior to the superior mesenteric vein (SMV). (d) Move the transducer further right to define the inferior vena cava (IVC). Ao, aorta.
Fig. 5. Method to trace the entire EHBD (inverse letter $C$ method). After defining the perihilar EHBD (arrow head) above the PV (A), rotate the transducer clockwise as if writing an inverse letter C (B) and (C); then, visualize the entire EHBD (arrow heads) (D).

PV, portal vein; IVC, inferior vena cava; EHBD, extrahepatic bile duct. Adapted from Okaniwa $\mathrm{S}$ and Iwashita K. Jpn J Med Ultrason 2018;45:471-480, with permission. ${ }^{44}$ due to overlying gas of the stomach (Fig. 6a). Move the transducer caudad and define the SMA and SV. Notice the pancreatic tail above the SV and LK (Fig. 6b). Adjust the focus deeper to get good images. Angle the transducer up and down over the pancreatic tail synchronizing with respiration to visualize the entire pancreatic tail. Then rock the transducer to the left side to trace the tips of pancreatic tail. As the pancreatic body to tail is slightly oblique from the lower right to the upper left, rotate the transducer to the same angle (Fig. 6c). At this point measure the diameter of MPD with magnified images.

Move the transducer to the right toward the pancreatic head (Fig. 6d). As the angle between the head and body is almost perpendicular, the transducer should be rotated from the transverse to the longitudinal section. After delineating the MPD and EHBD (Fig. 6e), move the transducer caudad to the transverse part of the duodenum. To detect PCIS, the MPD and accessory pancreatic duct should be traced with the high-resolution transducer with magnified images (Fig. 7).

As gas moves with the postural change, repeat this liver-tohead pass in both the supine and left lateral positions. In case of poor pancreatic head imaging, use the gallbladder as an echo window. After defining the gallbladder, move the transducer a little caudad rotating counterclockwise to visualize the EHBD and pancreatic head (Fig. 8).

\section{Left intercostal scanning}

As the pancreatic tail extends quite far posteriorly, scan the pancreatic tail not only from upper abdomen but from left intercostal (Fig. 9).

Place the transducer longitudinally on the left intercostal space, and define the LK. Move the transducer just a few intercostal spaces anteriorly angling a little upward, in order to see 

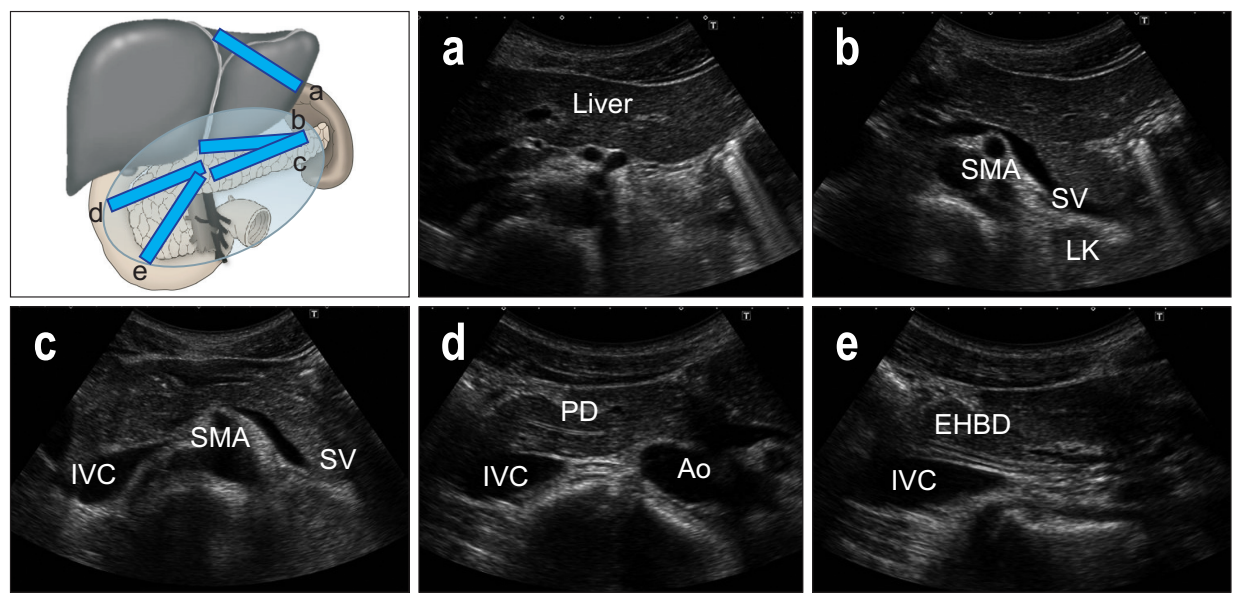

Fig. 6. Upper abdominal transverse scans. (a) Start scanning from the liver. (b) Notice the pancreatic tail above the SV and left LK. (c) As the pancreatic body to tail is slightly oblique from the lower right to the upper left, rotate the transducer to the same angle. (d) Move the transducer to the right toward the pancreatic head and magnify the image. (e) As the angle between the head and the body is almost perpendicular, the transducer should be rotated from the transverse to the longitudinal section.

SMA, superior mesenteric artery; SV, splenic vein; LK, left kidney; IVC, inferior vena cava; Ao, aorta; PD, pancreatic duct; EHBD, extrahepatic bile duct.
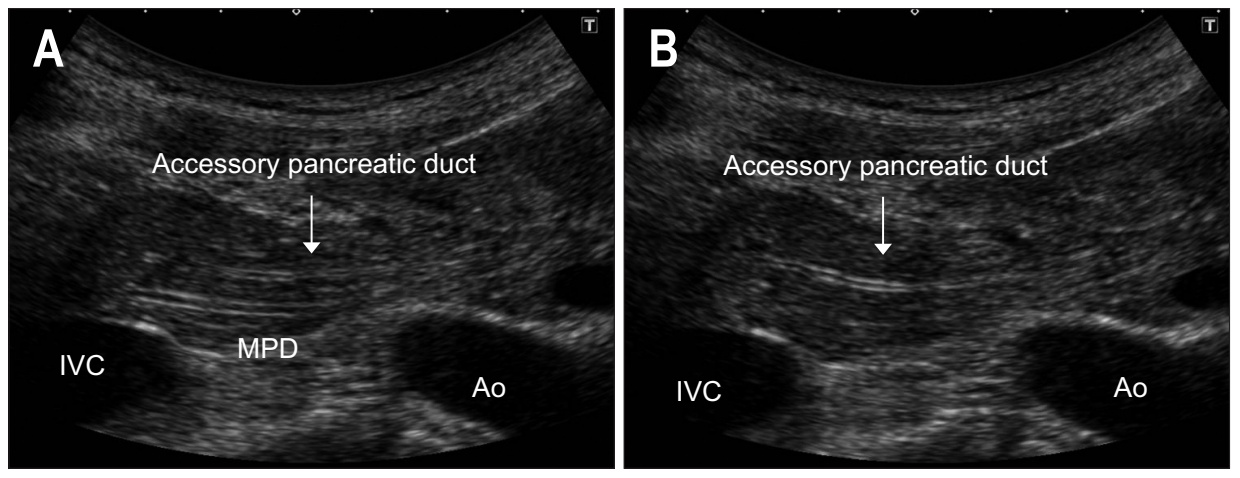

Fig. 7. Main pancreatic duct (MPD) and accessory pancreatic duct in the head of the pancreas. A highresolution transducer with magnified images can be used to trace the MPD (A) and accessory pancreatic duct precisely (B).

IVC, inferior vena cava; Ao, aorta.
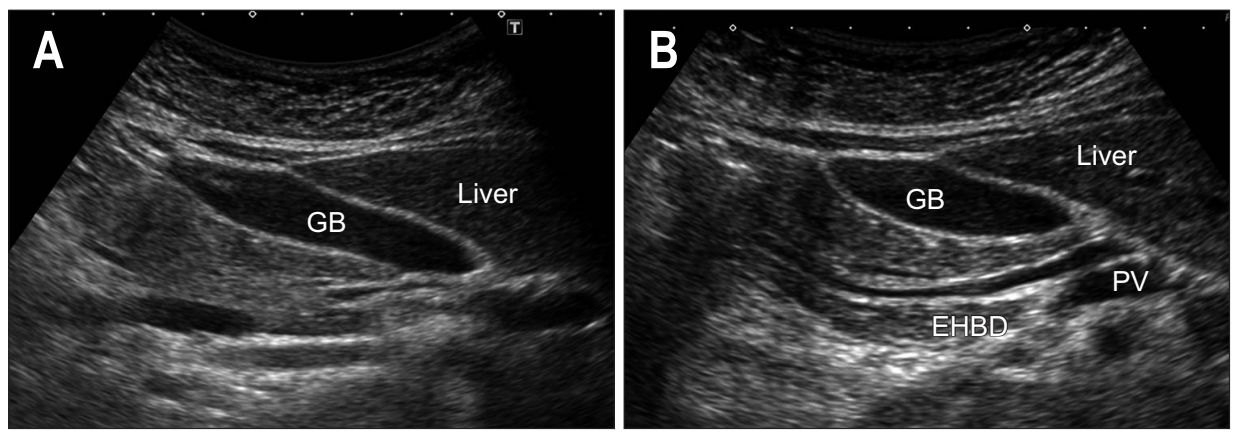

Fig. 8. (A, B) Scanning the pancreatic head through the gallbladder (GB). As the pancreatic head lies just beneath GB, use the GB as an acoustic window.

EHBD, extrahepatic bile duct; PV, portal vein.

the part of the pancreatic tail, which lies caudal to the SV. To scan the entire pancreatic tail, adjust the focus deeper and angle (tilt) the transducer in a fan-shaped pattern.

\section{PRACTICAL SOLUTIONS}

As positions of the pancreas change inside the body depending on posture, several different positions should be employed, such as the right lateral decubitus, sitting, and upright instead of only compressing strongly with the transducer. In cases with poor visualization instead of those postural changes, the liquidfilled stomach method with several positions is recommended.

Employing the liquid-filled stomach method in the Fowler position increases the detectability of pancreatic cysts $(92.2 \%)$ more than routine US in the supine position (70.2\%). ${ }^{7}$

\section{Right lateral decubitus position}

As normal pancreas has thin capsule and relatively little in- 

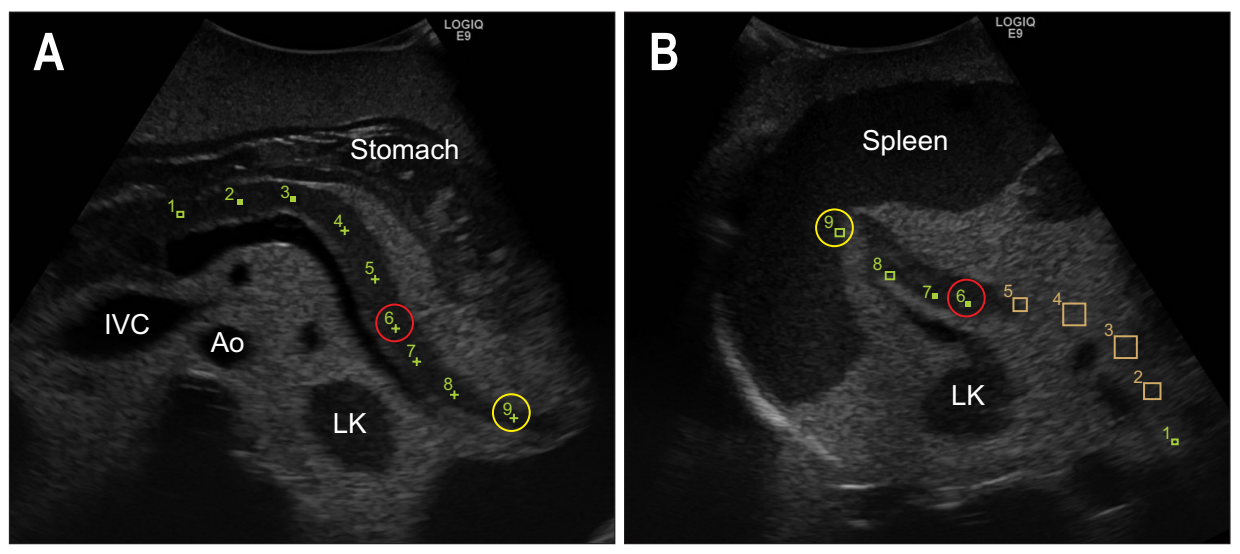

Fig. 9. Relationship between the transverse scan and left intercostal scan of the pancreatic tail in a phantom model. The navigation application shows the relationship between the upper abdominal transverse scan (A) and left intercostal scan (B). Middle pf the pancreatic tail (red circle), end of the pancreatic tail (yellow circle).

IVC, inferior vena cava; Ao, aorta; LK, left kidney.
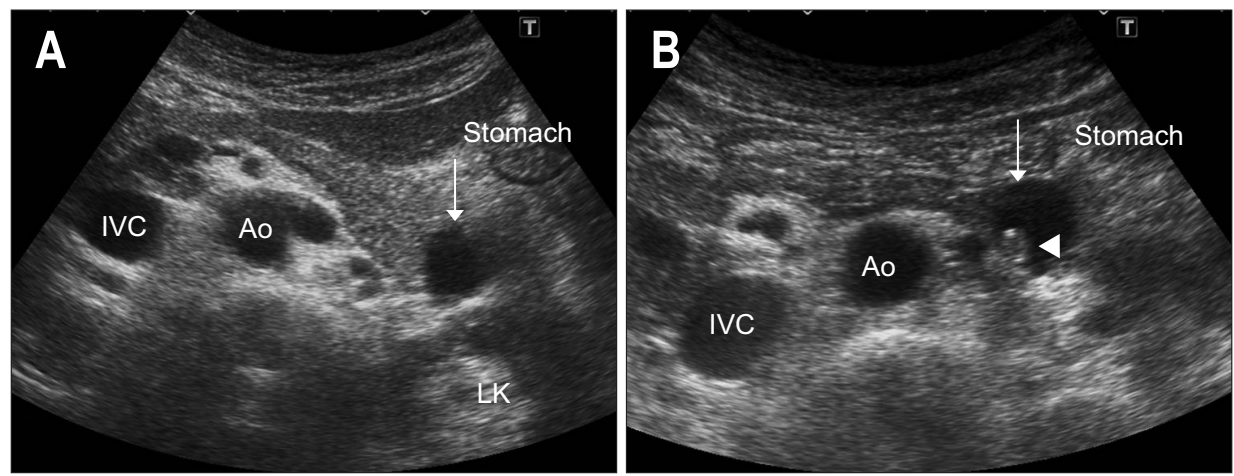

Fig. 10. The relationship between the supine position and right lateral decubitus position. (A) As the cyst in the tail (arrow) is located deeply dorsal in the supine position, precise visualization is difficult. (B) However, the tail moves into the shallower area in the right decubitus position, and the solid components (arrow head) inside the cystic lesion (arrow) can be visualized clearly.

IVC, inferior vena cava; Ao, aorta; LK, left kidney.


Fig. 11. Relationship between the supine position and Fowler position. (A) The pancreatic tail cannot be visualized clearly in the supine position. (B) After changing to the Fowler position, the liver and gastrointestinal tract descend slightly caudad, and visualization of the tail improves.

IVC, inferior vena cava; Ao, aorta; LK, left kidney.

ternal fibrous or muscular structure, both position and shape are determined partly by gravity and by the pressure of surrounding structures such as liver. After changing from the supine to the right decubitus position, some shift of the head towards the lower extremity of the right lobe of the liver, with apparent shortening of the body, and medial displacement of the tail is usually observed. ${ }^{45}$

As for HRI, the right lateral decubitus position should be employed to move the pancreatic tail into the shallower part of the abdomen. Lesions in the tail can be delineated with the highfrequency US transducer or linear probe in this position (Fig. 10).

\section{Fowler and sitting position}

As the liver and gastrointestinal tracts descend slightly down to caudad, and the liver serves as an acoustic window for the pancreas in the Fowler and sitting position, the visualization of the pancreas improves (Fig. 11).

Since patients should maintain this position without the stress and strain of abdominal muscle, an examination table with an adjustable backrest is preferred instead of patients placing their hands behind their back. ${ }^{7}$

Usually, the backrest is adjusted from approximately $30^{\circ}$ to $60^{\circ}$. This position is useful for elderly people to avoid having to 

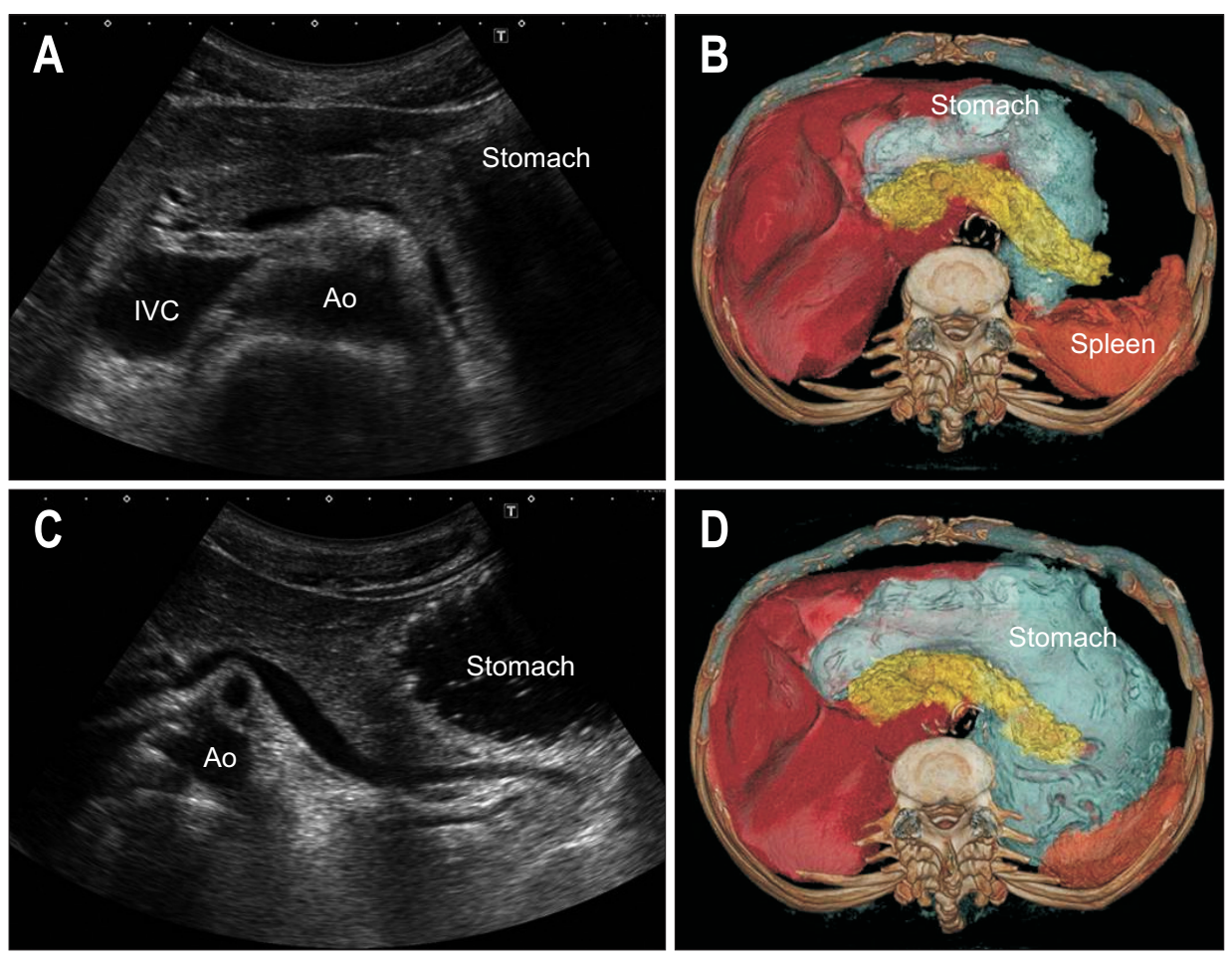

Fig. 12. Relationship between the supine position and liquid-filled stomach method in the semi-Fowler position. (A) Before ingestion of liquid, the pancreatic tail cannot be visualized clearly due to gas inside the stomach, and (B) computed tomography (CT) shows the space between the pancreatic tail and empty stomach. (C) After drinking water, the tail becomes clearly recognizable through the water-filled stomach and (D) CT shows the stomach close to the pancreatic tail.

IVC, inferior vena cava; Ao, aorta.

hold their breath. The left intercostal scanning at this position also improve longitudinal image of the pancreatic tail since detection depth is shallower. ${ }^{46}$

\section{Liquid-filled stomach method}

The water distention of the stomach and duodenum better outlines the anatomic boundaries and character of the pancreas. Crade et al. ${ }^{47}$ ask the patient to drink $300 \mathrm{~mL}$ of tap water in the right decubitus position using a straw to minimize amount of swallowed air. Following imbibition change the position from the supine to the slight right-side-down decubitus, and the severe right-side-down decubitus position to move the water from the stomach body, antrum, to the duodenum, and scan the tail, body, and head, respectively.

Patients may also drink 200 to $300 \mathrm{~mL}$ water or tea at the sitting position without a straw. Air bubbles may be observed, but soon dissipate. Start scanning for the tail and body in the supine or Fowler position with the right anterior oblique position. However, as the pancreatic tail still locates deeply dorsal in the Fowler and sitting position, the image of pancreatic tail cannot be visualized precisely in some cases. In this situation, the right decubitus position using high resolution transducer with magnified image may be useful for the precise visualization not only for the tail but also the head (Fig. 12).

If these maneuvers fail to permit visualization of the pancreas, scan the patient at the upright position, thereby bringing the liver down as low as possible. ${ }^{48}$

\section{CONCLUSIONS}

Understanding of the US findings of PC and HRI and adopting various maneuvers will enable us to visualize a wider range of pancreas and detect PC in its early stages.

\section{CONFLICTS OF INTEREST}

No potential conflict of interest relevant to this article was reported.

\section{ORCID}

Shinji Okaniwa https://orcid.org/0000-0001-6718-6901

\section{REFERENCES}

1. Kamisawa T, Wood LD, Itoi T, Takaori K. Pancreatic cancer. Lancet 2016;388:73-85.

2. Tanaka S, Kitamra T, Yamamoto K, et al. Evaluation of routine sonography for early detection of pancreatic cancer. Jpn J Clin Oncol 1996;26:422-427.

3. Kanno A, Masamune A, Hanada K, et al. Multicenter study of early pancreatic cancer in Japan. Pancreatology 2018;18:61-67.

4. Yamaguchi K, Okusaka T, Shimizu K, et al. Clinical practice guidelines for pancreatic cancer 2016 from the Japan Pancreas Society: a synopsis. Pancreas 2017;46:595-604.

5. Tanaka S, Nakaizumi A, Ioka T, et al. Periodic ultrasonography checkup for the early detection of pancreatic cancer: preliminary 
report. Pancreas 2004;28:268-272.

6. Tanaka S, Nakao M, Ioka T, et al. Slight dilatation of the main pancreatic duct and presence of pancreatic cysts as predictive signs of pancreatic cancer: a prospective study. Radiology 2010;254:965-972.

7. Nakao M, Katayama K, Fukuda J, et al. Evaluating the ability to detect pancreatic lesions using a special ultrasonography examination focusing on the pancreas. Eur J Radiol 2017;91:10-14.

8. Siegel R, Ma J, Zou Z, Jemal A. Cancer statistics, 2014. CA Cancer J Clin 2014;64:9-29.

9. Jemal A, Bray F, Center MM, Ferlay J, Ward E, Forman D. Global cancer statistics. CA Cancer J Clin 2011;61:69-90.

10. Yachida S, Jones S, Bozic I, et al. Distant metastasis occurs late during the genetic evolution of pancreatic cancer. Nature 2010;467:1114-1117.

11. Egawa S, Toma H, Ohigashi H, et al. Japan Pancreatic Cancer Registry; 30th year anniversary: Japan Pancreas Society. Pancreas 2012;41:985-992.

12. Haeno H, Gonen M, Davis MB, Herman JM, Iacobuzio-Donahue CA, Michor F. Computational modeling of pancreatic cancer reveals kinetics of metastasis suggesting optimum treatment strategies. Cell 2012;148:362-375.

13. Hruban RH, Adsay NV, Albores-Saavedra J, et al. Pancreatic intraepithelial neoplasia: a new nomenclature and classification system for pancreatic duct lesions. Am J Surg Pathol 2001;25:579586.

14. Iiboshi T, Hanada K, Fukuda T, Yonehara S, Sasaki T, Chayama $\mathrm{K}$. Value of cytodiagnosis using endoscopic nasopancreatic drainage for early diagnosis of pancreatic cancer: establishing a new method for the early detection of pancreatic carcinoma in situ. Pancreas 2012;41:523-529.

15. Takaori K, Matsusue S, Fujikawa T, et al. Carcinoma in situ of the pancreas associated with localized fibrosis: a clue to early detection of neoplastic lesions arising from pancreatic ducts. Pancreas 1998:17:102-105.

16. Maehira H, Sugiura T, Kanemoto H, et al. A case of carcinoma in situ of the pancreas with a surrounding fibrotic region. Suizo 2014;29:919-925.

17. Shindo H, Fukasawa M, Takano S, et al. A case of carcinoma in situ of the pancreas concomitant with branch duct intraductal papillary mucinous neoplasm. Suizo 2014;29:742-748.

18. Mizutani Y, Otsuka H, Morishima H, Fujitsuka Y, Katayama M, Ishikawa $\mathrm{H}$. A case of carcinoma in situ of the pancreas. Suizo 2013;28:785-791.

19. Seki M, Ninomiya E, Takano K, et al. Pancreatogram findings for carcinoma in situ (CIS) of the pancreas seen on endoscopic retrograde cholangiopancreatography and postoperative pancreatography of resected specimens: can CIS be diagnosed preoperatively? Pancreatology 2008;8:142-152.

20. Hanada K, Okazaki A, Hirano N, et al. Diagnostic strategies for early pancreatic cancer. J Gastroenterol 2015;50:147-154.

21. Terminology and Diagnostic Criteria Committee, Japan Society of Ultrasonics in Medicine. Manual for abdominal ultrasound in cancer screening and health checkups. Jpn J Med Ultrason 2015;42:201-224.

22. Doust BD, Pearce JD. Gray-scale ultrasonic properties of the normal and inflamed pancreas. Radiology 1976;120:653-657.

23. Haber K, Freimanis AK, Asher WM. Demonstration and dimensional analysis of the normal pancreas with gray-scale echography. AJR Am J Roentgenol 1976;126:624-628.

24. Terminology and Diagnostic Criteria Committee, Japan Society of Ultrasonics in Medicine. Ultrasonographic diagnostic criteria for pancreatic cancer. J Med Ultrason (2001) 2013;40:497-504.

25. Kobayashi S, Hasuo S, Miyakoshi M, et al. Ultrasonographic findings of 46 resected cases with invasive ductal carcinoma of the pancreas within $20 \mathrm{~mm}$ in size. Jpn J Med Ultrasoonic 2018;45: 301-309.

26. Laffan TA, Horton KM, Klein AP, et al. Prevalence of unsuspected pancreatic cysts on MDCT. AJR Am J Roentgenol 2008;191:802807.

27. de Jong K, Nio CY, Hermans JJ, et al. High prevalence of pancreatic cysts detected by screening magnetic resonance imaging examinations. Clin Gastroenterol Hepatol 2010;8:806-811.

28. Soroida Y, Sato M, Hikita H, et al. Pancreatic cysts in general population on ultrasonography: prevalence and development of risk score. J Gastroenterol 2016;51:1133-1140.

29. Okaniwa S, Iwashita K. Macroscopic findings and ultrasound differential diagnosis of pancreatic cystic lesions. Jpn J Med Ultrason 2017;44:235-244.

30. Tanaka M, Chari S, Adsay V, et al. International consensus guidelines for management of intraductal papillary mucinous neoplasms and mucinous cystic neoplasms of the pancreas. Pancreatology 2006;6:17-32.

31. Itai Y, Moss AA, Goldberg HI. Pancreatic cysts caused by carcinoma of the pancreas: a pitfall in the diagnosis of pancreatic carcinoma. J Comput Assist Tomogr 1982;6:772-776.

32. Canto MI, Hruban RH, Fishman EK, et al. Frequent detection of pancreatic lesions in asymptomatic high-risk individuals. Gastroenterology 2012;142:796-804.

33. Tanaka S, Nakaizumi A, Ioka T, et al. Main pancreatic duct dilatation: a sign of high risk for pancreatic cancer. Jpn J Clin Oncol 2002;32:407-411.

34. Ikeda M, Sato T, Morozumi A, et al. Morphologic changes in the pancreas detected by screening ultrasonography in a mass survey, with special reference to main duct dilatation, cyst formation, and calcification. Pancreas 1994;9:508-512.

35. Wachsberg RH. Respiratory variation of the diameter of the pancreatic duct on sonography. AJR Am J Roentgenol 2000;175:1459-1461.

36. Ishikawa 0 , Ohigashi $\mathrm{H}$, Imaoka S, et al. Minute carcinoma of the pancreas measuring $1 \mathrm{~cm}$ or less in diameter: collective review of Japanese case reports. Hepatogastroenterology 1999;46:8-15.

37. Siegel MJ, Martin KW, Worthington JL. Normal and abnormal pancreas in children: US studies. Radiology 1987;165:15-18. 
38. Sirli R, Sporea I. Ultrasound examination of the normal pancreas. Med Ultrason 2010;12:62-65.

39. Takahashi S, Akita K, Goseki N, Sato T. Spatial arrangement of the pancreatic ducts in the head of the pancreas with special reference to the branches of the uncinate process. Surgery 1999;125:178185.

40. Pirri C, Cui XW, De Molo C, Ignee A, Schreiber-Dietrich DG, Dietrich CF. The pancreatic head is larger than often assumed. Z Gastroenterol 2013;51:390-394.

41. Sato M, Ishida H, Konno K, et al. Pancreatic uncinate carcinoma: sonographic findings. Abdom Imaging 2001;26:64-68.

42. Gabata T, Kadoya M, Terayama N, Sanada J, Kobayashi S, Matsui

0. Groove pancreatic carcinomas: radiological and pathological findings. Eur Radiol 2003;13:1679-1684.

43. Sumi H, Itoh A, Kawashima H, et al. Preliminary study on evaluation of the pancreatic tail observable limit of transabdominal ultrasonography using a position sensor and CT-fusion image. Eur
J Radiol 2014;83:1324-1331.

44. Okaniwa S, Iwashita K. How should we evaluate the pancreatobiliary system?: pitfalls and practical solutions. Jpn J Med Ultrason 2018;45:471-480.

45. Gimlette TM. Change in shape and position of the pancreas with posture of the patient. Clin Nucl Med 1979;4:111-115.

46. Tsuruoka N, Kanada S, Takeuchi K. The ultrasonographic scanning techniques and detect ability of the cystic lesions in the pancreatic body and tail by posture conversion. Jpn J Med Ultrasound Technol 2005;30:4-8.

47. Crade M, Taylor KJ, Rosenfeld AT. Water distention of the gut in the evaluation of the pancreas by ultrasound. AJR Am J Roentgenol 1978;131:348-349.

48. Jacobson P, Crade M, Taylor KJ. The upright position while giving water for the evaluation of the pancreas. J Clin Ultrasound 1978;6:353-354. 